Published in final edited form as:

Science. 2020 June 05; 368(6495): 1122-1127. doi:10.1126/science.aax4040.

\title{
PIRs mediate innate myeloid cell memory to nonself MHC molecules
}

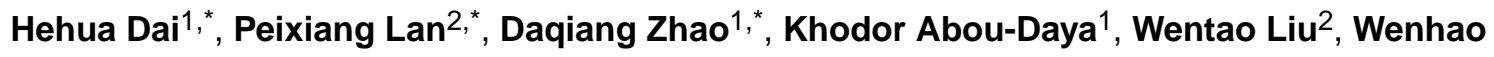 \\ Chen $^{2}$, Andrew J. Friday ${ }^{1}$, Amanda L. Williams ${ }^{1}$, Tao Sun ${ }^{3}$, Jianjiao Chen ${ }^{3}$, Wei Chen ${ }^{3,4}$, \\ Steven Mortin-Toth ${ }^{5}$, Jayne S. Danska ${ }^{5,6}$, Chris Wiebe ${ }^{7}$, Peter Nickerson ${ }^{7,8}$, Tengfang Li ${ }^{1}$, \\ Lisa R. Mathews ${ }^{1}$, Hêth R. Turnquist ${ }^{1,9}$, Matthew L. Nicotra ${ }^{1,9,10}$, Sebastien Gingras ${ }^{9}$, Eiji \\ Takayama $^{11}$, Hiromi Kubagawa ${ }^{12}$, Mark J. Shlomchik ${ }^{9}$, Martin H. Oberbarnscheidt ${ }^{1,9,13, \dagger}$, \\ Xian C. Li ${ }^{2}, \dagger$, Fadi G. Lakkis ${ }^{1,9,14, \dagger}$ \\ ${ }^{1}$ Thomas E. Starzl Transplantation Institute and Department of Surgery, University of Pittsburgh, \\ Pittsburgh, PA, USA. \\ ${ }^{2}$ Immunobiology and Transplant Science Center, Houston Methodist Research Institute, Houston \\ TX, and Department of Surgery, Weill Cornell Medical College of Cornell University, New York, \\ NY, USA. \\ ${ }^{3}$ Department of Pediatrics, University of Pittsburgh, PA, USA. \\ ${ }^{4}$ Department of Biostatistics, University of Pittsburgh, PA, USA. \\ ${ }^{5}$ Program in Genetics and Genome Biology, Hospital for Sick Children Research Institute, Toronto, \\ Ontario, Canada. \\ ${ }^{6}$ Departments of Immunology and Medical Biophysics, University of Toronto, Ontario, Canada \\ ${ }^{7}$ Department of Medicine, University of Manitoba, Winnipeg, Manitoba, Canada. \\ ${ }^{8}$ Department of Immunology, University of Manitoba, Winnipeg, Manitoba, Canada. \\ ${ }^{9}$ Department of Immunology, University of Pittsburgh, PA, USA.
}

\footnotetext{
†Correspondence to: mho6@pitt.edu; xcli@HoustonMethodist.org; lakkisf@upmc.edu. Authors contributions:

H.D., P.L., D.Z., W.L., W.C., A.J.F., T.L., and L.R.M. performed experiments and analyzed data. K.A-D., T.S, and J.C. performed bioinformatic analysis. W.C. supervised bioinformatic analysis. A.L.W. and A.M-T. performed mouse breeding and genotyping. J.S.D. supervised generation and genotyping of NOD congenic mice and edited manuscript. C.W. and P.N. provided data and insights into human correlates of mouse findings. M.L.N. performed comparative mouse genomics and edited manuscript. S.G. designed and constructed gene knockout mice and contributed to manuscript writing. E.T. and H.K. designed, constructed, and provided expression vectors and reagents and edited manuscript. M.J.S. supervised design and construction of Pir ${ }^{-1-}$ mice, provided the mice, and edited manuscript. M.H.O. participated in experimental design, coordinated experiments, trained and supervised personnel, and wrote the manuscript. X.C.L and F.G.L. conceived the idea, designed experiments, analyzed data, supervised overall work, and wrote the manuscript.

*These authors contributed equally.

Publisher's Disclaimer: This manuscript has been accepted for publication in Science. This version has not undergone final editing. Please refer to the complete version of record at https://www.sciencemag.org. The manuscript may not be reproduced or used in any manner that does not fall within the fair use provisions of the Copyright Act without the prior, written permission of AAAS.

Competing interests:

None.

Data and materials availability:

Raw scRNA-seq data have been deposited into Gene Expression Omnibus (GEO) database (accession number: ). All other data needed to evaluate the conclusions of the paper are available in the manuscript or the supplementary materials.
} 
${ }^{10}$ Center for Evolutionary Biology \& Medicine (CEBaM), University of Pittsburgh, PA, USA.

${ }^{11}$ Department of Oral Biochemistry, Asahi University School of Dentistry.

${ }^{12}$ Humoral Immune Regulation, Deutsches Rheuma-Forschungszentrum (DRFZ), Berlin, Germany.

${ }^{13}$ Center for Critical Care Nephrology, Department of Critical Care Medicine, University of Pittsburgh, PA, USA.

${ }^{14}$ Department of Medicine, University of Pittsburgh, PA, USA.

\section{Abstract}

Immunological memory specific to previously encountered antigens is a cardinal feature of adaptive lymphoid cells. It is unknown however whether innate myeloid cells retain memory of prior antigenic stimulation and respond to it more vigorously upon subsequent encounters. Here, we show that murine monocytes and macrophages acquire memory specific to MHC-I antigens and identify paired immunoglobulin-like receptors-A (PIR-A) as the MHC-I receptors necessary for the memory response. We demonstrate that deleting PIR-A in the recipient or blocking PIR-A binding to donor MHC-I molecules blocks memory and attenuates kidney and heart allograft rejection. Thus, innate myeloid cells acquire alloantigen-specific memory that can be targeted to improve transplant outcomes.

\section{One Sentence Summary:}

Targeting monocyte and macrophage receptors that detect MHC antigens blocks innate immune memory and attenuates transplant rejection

Immunological memory protects the host against infection but is also a potent barrier to transplant survival (1). Initially thought to be confined to $\mathrm{T}$ and $\mathrm{B}$ lymphocytes, it is now evident that innate lymphoid (NK) and myeloid cells also acquire features of immunological memory. In lymphoid cells, memory responses are anamnestic (1-3). By contrast, myeloid cells exhibit memory-like responses dubbed "trained immunity" as secondary stimuli that elicit enhanced reactions need not be related to the primary stimulus (4). Whether myeloid cells acquire memory specific to previously encountered antigens is not known. Given the key roles of myeloid cells in host defense (5), answering this question is paramount.

We studied monocyte and macrophage reactions to allogeneic bone marrow plug grafts placed under the kidney capsule. Monocytes and macrophages sense MHC and non-MHC determinants on allogeneic tissues and mature to antigen-presenting, inflammatory, or cytotoxic cells (6-9). To investigate memory, B6- $\mathrm{Rag}^{-1-} \mathrm{II2 \textrm {rg } ^ { - 1 }}$ mice, which lack B, T, NK, and innate lymphoid cells, were immunized with irradiated allogeneic splenocytes. They were then re-challenged with grafts from same-party (same source as the immunizing splenocytes) or third-party allogeneic donors after 7, 28 or 49 days. B6- $\mathrm{Rag}^{-/-} I 12 \mathrm{rg}^{-/-}$ (H-2b) mice immunized with BALB/c (H-2d) splenocytes and re-challenged with sameparty $\mathrm{BALB} / \mathrm{c}$ allografts 7 or 28 days later exhibited significantly greater graft infiltration with recipient monocyte-derived dendritic cells (Mo-DCs) than unimmunized recipients or those immunized with syngeneic (B6) or third-party allogeneic $(\mathrm{C} 3 \mathrm{H}, \mathrm{H}-2 \mathrm{k})$ splenocytes 
(Fig. 1A). This enhanced response dissipated by 49 days after immunization. B6- $\mathrm{Rag}^{-/-}$II2rg -/- recipients of $\mathrm{C} 3 \mathrm{H}$ grafts mounted a heightened response if previously immunized with $\mathrm{C} 3 \mathrm{H}$ but not B6 or BALB/c splenocytes, further demonstrating allospecificity (Fig. 1B). Enhanced responsiveness could not be attributed to immunogen persistence because neither donor splenocytes nor intact donor MHCI molecules were detected in the host's circulation or spleen at the time of re-challenge (fig. S1A). Thus, host monocytes appear to acquire specific memory to previously encountered alloantigens.

To rule out any possible contribution of lymphoid cells in the graft, we repeated the experiments using $\mathrm{Rag}^{-1-} I 12 \mathrm{rg}^{-1-}$ donors. B6- $\mathrm{Rag}^{-1-} \mathrm{Il} 2 \mathrm{rg}^{-1-}$ recipients responded more vigorously to BALB/c- $\mathrm{Rag}^{-1-} I 12 \mathrm{rg}^{-/-}$bone marrow plug allografts if previously immunized with BALB/c- $\mathrm{Rag}^{-1-} I 12 \mathrm{rg}^{-/-}$but not NOD- $\mathrm{Rag}^{-/} I I 2 \mathrm{rg}^{-1-}$ splenocytes (Fig. 1C), whereas NOD- $\mathrm{Rag}^{-/-} I 12 \mathrm{rg}^{-/-}$immunization enhanced the response to NOD- $\mathrm{Rag}^{-/-} I 12 \mathrm{rg}^{-/-}$allografts (Fig. 1D). Thus, monocyte memory to alloantigen is not dependent on lymphoid cells from either the graft or the recipient.

The primary innate alloresponse that generates Mo-DCs is mediated by inflammatory $\left(\right.$ Ly6C ${ }^{\text {hi }}$ ) monocytes $(6,7)$. To test whether this monocyte subset acquires memory, we sorted Ly6C ${ }^{\text {hi }}$ monocytes from B6- $\mathrm{Rag}^{-/-} \mathrm{II} \mathrm{rg}^{-/-}$mice 7, 21, or 35 days after immunization and transferred them to naive B6- $\mathrm{Rag}^{-/-} \mathrm{II2 \textrm {rg } ^ { - / }}$ hosts (Fig. 1E). Mice that received monocytes from allostimulated donors mounted a significantly greater response to BALB/c allografts than those that received monocytes from donors stimulated with syngeneic cells. This demonstrates that Ly6 $\mathrm{C}^{\text {hi }}$ monocytes are capable of mediating memory and that they retain memory function up to several weeks after their first encounter with alloantigen.

Macrophages acquire the ability to kill allogeneic targets if first challenged with allogeneic cells along with CD40 receptor cross-linking (9). We therefore examined whether macrophages also acquire allospecific memory. B6- $\mathrm{Rag}^{-/-} I 12 \mathrm{rg}^{-1-}$ mice were left unstimulated (naive) or were immunized with BALB/c splenocytes plus agonistic anti-CD40 antibody and an in vivo killing assay was performed 14 and 28 days later (Fig. 1F). BALB/cimmunized mice killed BALB/c but not syngeneic (B6) or third-party $(\mathrm{C} 3 \mathrm{H})$ targets, suggesting that macrophages, like monocytes, acquire memory specific to previously encountered alloantigens.

MHC proteins are the principal alloantigens in humans and mice. To test whether monocyte/ macrophage memory is dependent on detection of nonself $\mathrm{MHC}$ molecules, we immunized B6- $\mathrm{Rag}^{-/-} \mathrm{II} 2 \mathrm{rg}^{-/-}(\mathrm{H}-2 \mathrm{~b})$ recipients with irradiated BALB/c (H-2d) splenocytes and rechallenged them with BALB.B (H-2b) grafts. BALB.B and B6 mice share the same MHC (H-2) but differ at non-MHC loci. Immunization with BALB/c cells did not enhance the response to BALB.B grafts, indicating that monocyte memory is against nonself MHC molecules (Fig. 1G). The same was true for macrophages (Fig. 1H). Moreover, BALB/cimmunized hosts killed b2-microglobulin knockout targets less efficiently than wildtype cells, indicating that the response is primarily to nonself MHC-I molecules (Fig. $1 \mathrm{H})$. Furthermore, macrophages from BALB/c-immunized B6 mice bound allogeneic H-2Dd (and to some extent allogeneic $\mathrm{H}-2 \mathrm{Kd}$ ) but not syngeneic $\mathrm{H}-2 \mathrm{Db}$ and $\mathrm{H}-2 \mathrm{~Kb}$ MHC-I tetramers (Fig. 1I). Additionally, host exposure to both MHC and non-MHC polymorphisms at the 
time of priming was necessary to generate memory (fig. S1B). Polymorphism in the nonMHC signal regulatory protein-alpha (Sirpa) gene, which is known to trigger the primary monocyte alloresponse (8), was required.

A database search across 17 mouse genomes for potential MHC-I-binding molecules on myeloid cells identified several families of polymorphic immunoglobulin ( $\mathrm{Ig}$ ) superfamily receptors. Among these were the paired Ig-like receptors (PIRs), orthologs of human leukocyte Ig-like receptors (LILRs) (10). Closely linked genes coding for six PIR-A and one PIR-B protein are located on mouse chromosome 7 (11). PIR-B is non-polymorphic and binds a wide spectrum of MHC-I molecules (12). Its cytosolic domain transmits inhibitory signals $(12,13)$, whereas PIR-As are stimulatory $(14,15)$. PIR-A isoforms possibly bind distinct MHC-I molecules (12), and PIR-A expression is upregulated upon myeloid cell differentiation and activation (15). Using anti-PIR-A/B and anti-PIR-B antibodies, we detected PIR-A expression on resting Ly6 $\mathrm{C}^{\text {hi }}$ monocytes (Fig. 2A). Macrophages expressed both PIR-A and PIR-B and upregulated PIR-A after allostimulation (Fig. 2B). Blocking these receptors with an anti-PIRA/B antibody inhibited monocyte memory in Pirb-sufficient

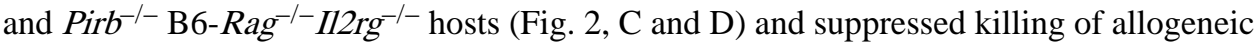
targets in alloimmunized B6- $\mathrm{Rag}^{-/-} I 12 \mathrm{rg}^{-/-}$mice (Fig. 2E). We confirmed these results by treating animals with PIR-A3/Fc fusion protein, which preferentially blocks PIR-A3 from binding to its MHC-I ligand, H-2Dd. The preferred ligand of PIR-A3 is H-2Dd (Fig. 2, F to H). H2-Dd tetramers triggered the strongest signal in reporter cells expressing PIR-A3 (Fig. 2F). They bound PIR-A3-transfected 3T3 fibroblasts better than H-2Db tetramers (Fig. 2G) and binding of PIR-A3/Fc was greater to BALB/c $\left(\mathrm{H}-2 \mathrm{Dd}^{+}\right)$than $\mathrm{B} 6$ cells $\left(\mathrm{H}-2 \mathrm{Db}^{+}\right.$but $\mathrm{H}^{-2 \mathrm{Dd}^{-}}$) (Fig. 2H). Indeed, PIR-A3/Fc inhibited both macrophage (Fig. 2I) and monocyte (Fig. 2J) memory in BALB/c-immunized B6- $\mathrm{Rag}^{-/-} I 12 \mathrm{rg}^{-/-}$mice. By contrast, PIR-A1/Fc did not inhibit the killing of BALB/c (H-2d) targets (Fig. 2I). Furthermore, PIR-A3/Fc failed to suppress the monocyte memory response to $\mathrm{C} 3 \mathrm{H}(\mathrm{H}-2 \mathrm{k})$ grafts (Fig. 2J), confirming the specificity of PIR-A3 to BALB/c H-2Dd molecules. Finally, we generated PIR-A locus knockout ( Pira $^{-/}$) mice lacking PIR-A1-5 (fig. S2), which were bred onto the B6-Rag ${ }^{-1} \mathrm{II}_{2 \mathrm{rg}^{-1}}$ background. Strikingly, these mice did not mount a monocyte memory response to BALB/c allografts 7 or 28 days after priming (Fig. 2K). By contrast, the primary response to BALB/c allografts (Fig. 2K) and primary DC and macrophage functions (antigen presentation and IL-6 production) (fig. S3) were not inhibited in Pira $^{-/-}$recipients. Thus, PIR-A mediates innate memory.

We next investigated how monocyte memory is acquired and whether it resembles NK memory (16). B6 mice immunized with BALB/c (H-2d) cells selectively increased the proportion and number of splenic monocytes that bind the $\mathrm{H}-2 \mathrm{Dd}$ tetramer, whereas $\mathrm{C} 3 \mathrm{H}$ (H-2k) immunization exclusively increased H-2Dk-binding cells (Fig. 3A and fig. S4A). This effect was preserved in Pirb ${ }^{-/-}$but abolished in Pira ${ }^{-1-}$ mice. Only a minority of monocytes bound more than one type of tetramer (fig. S4B), suggesting that PIR-A expression is variegated. Single-cell RNA sequence (scRNA-seq) analysis of splenic monocytes 1 and 4 weeks after immunization showed that monocytes followed a pseudotime trajectory from a "starting" state $(\mathrm{S})$, which dominated in mice stimulated with syngeneic cells, to "expanding" states (E1-3), which increased proportionally in allostimulated groups (Fig. 3, B and C). E states were enriched for cell cycle, antigen presentation, and immune 
pathways such as allograft rejection and graft-versus-host-disease (Data S1-2); and exhibited increased Pira and reduced Pirb expression (Fig. 4D). Cell cycle pathway enrichment is consistent with prior evidence of monocyte proliferation post-allostimulation (8). Finally, monocytes that expressed mRNA of two different Pira alleles constituted a small minority before and after allostimulation (Fig. 4E), providing further evidence for variegated PIR-A expression. Thus, a possible mechanism of monocyte memory is the clonal expansion of cells specific to a particular nonself MHC molecule.

Mo-DCs and macrophages contribute to allograft rejection in mice and humans (7, 17-19). It is unknown however whether monocyte-macrophage memory mediated by PIR-A plays a role in rejection. Kidney allografts survived long-term ( $>125$ days) and maintained normal serum creatinine in lymphoid cell-sufficient wildtype and $\mathrm{Pira}^{-/-}$recipients but were rapidly rejected by Pirb ${ }^{-1}$ mice (Fig. 4, A and B), consistent with the inhibitory functions of PIR-B 12, 13). PIR-A3/Fc prevented acute allograft rejection in Pirb ${ }^{-1}$ mice (Fig. 4, A and B), suggesting that PIR-A accelerates rejection in the absence of PIR-B. Chronic rejection and cellular infiltrates in wildtype mice were significantly attenuated in Pira $^{-/-}$mice and in Pirb ${ }^{-/-}$recipients treated with PIR-A3/Fc (Fig. 4, C and D, and fig. S5A). As expected, chronic rejection did not occur in grafts that were rejected acutely $\left(\right.$ Pirb $^{-/-}$and Pirb ${ }^{-/}+\mathrm{Ctrl}$ IgG groups, Fig. 4, C and D). We also observed a pathogenic role for PIR-A in heart transplantation. CTLA4-Ig or PIR-A3/Fc alone caused significant but modest prolongation of allograft survival, whereas their combined administration markedly extended survival and prevented pathology associated with acute or chronic rejection (Fig. 4, E and F, and fig. S5, B and C). Neither PIR-A deficiency nor blockade suppressed alloantibody production (fig. S5, D and E). Thus, PIR-A, which is necessary for monocyte and macrophage memory, promotes allograft rejection whereas PIR-B tempers it.

The findings described here identify a pathway of MHC-I allorecognition that is mediated by monocytes and macrophages, generates memory specific to previously encountered MHC-I alloantigens, and contributes to allograft rejection. This pathway extends the domain of classical immunological memory to innate myeloid cells and can be potentially targeted to improve the survival of life-saving organ transplants.

\section{Supplementary Material}

Refer to Web version on PubMed Central for supplementary material.

\section{Acknowledgments:}

We would like to thank C. Bi and Z. Kou of the Transgenic and Gene Targeting Core (Department of Immunology, Univ. of Pittsburgh School of Medicine) for microinjection of zygotes and production of Pira and Pirb mutant mice.

Funding:

This work was supported by NIH grants AI145881 (M.H.O.), AI080779 (X.C.L.), and AI099465 (F.G.L); and funds from the Frank \& Athena Sarris Chair in Transplantation Biology at the University of Pittsburgh (F.G.L.). 


\section{References and Notes:}

1. Valujskikh A, Lakkis FG, In remembrance of things past: memory T cells and transplant rejection. Immunol Rev 196, 65-74 (2003). [PubMed: 14617198]

2. O'Leary JG, Goodarzi M, Drayton DL, von Andrian UH, T cell- and B cell-independent adaptive immunity mediated by natural killer cells. Nat Immunol 7, 507-516 (2006). [PubMed: 16617337]

3. Sun JC, Beilke JN, Lanier LL, Adaptive immune features of natural killer cells. Nature 457, 557561 (2009). [PubMed: 19136945]

4. Netea MG et al., Trained immunity: A program of innate immune memory in health and disease. Science 352, aaf1098(2016). [PubMed: 27102489]

5. Bassler K, Schulte-Schrepping J, Warnat-Herresthal S, Aschenbrenner AC, Schultze JL, The myeloid cell compartment-cell by cell. Annu Rev Immunol 37, 269-293 (2019). [PubMed: 30649988]

6. Zecher D, van Rooijen N, Rothstein D, Shlomchik W, Lakkis F, An innate response to allogeneic nonself mediated by monocytes. J Immunol 183, 7810-7816 (2009). [PubMed: 19923456]

7. Oberbarnscheidt MH et al., Non-self recognition by monocytes initiates allograft rejection. J Clin Invest 124, 3579-3589 (2014). [PubMed: 24983319]

8. Dai $\mathrm{H}$ et al., Donor SIRPa polymorphism modulates the innate immune response to allogeneic grafts. Science Immunology 2, eaam6202(2017). [PubMed: 28783664]

9. Liu W, Xiao X, Demirci G, Madsen J, Li XC, Innate NK cells and macrophages recognize and reject allogeneic nonself in vivo via different mechanisms. J Immunol 188, 2703-2711 (2012). [PubMed: 22327074]

10. Takai T, Paired immunoglobulin-like receptors and their MHC class I recognition. Immunology 115, 433-440 (2005). [PubMed: 16011512]

11. Kubagawa H, Burrows PD, Cooper MD, A novel pair of immunoglobulin-like receptors expressed by B cells and myeloid cells. Proc Natl Acad Sci U S A 94, 5261-5266 (1997). [PubMed: 9144225]

12. Nakamura A, Kobayashi E, Takai T, Exacerbated graft-versus-host disease in Pirb-/-mice. Nat Immunol 5, 623-629 (2004). [PubMed: 15146181]

13. Ma G et al., Paired immunoglobin-like receptor-B regulates the suppressive function and fate of myeloid-derived suppressor cells. Immunity 34, 385-395 (2011). [PubMed: 21376641]

14. Maeda A, Kurosaki M, Kurosaki T, Paired immunoglobulin-like receptor (PIR)-A is involved in activating mast cells through its association with Fc receptor gamma chain. J Exp Med 188, 991995 (1998). [PubMed: 9730901]

15. Kubagawa $\mathrm{H}$ et al., Biochemical nature and cellular distribution of the paired immunoglobulin-like receptors, PIR-A and PIR-B. J Exp Med 189, 309-318 (1999). [PubMed: 9892613]

16. Nabekura T, Lanier LL, Antigen-specific expansion and differentiation of natural killer cells by alloantigen stimulation. J Exp Med 211, 2455-2465 (2014). [PubMed: 25366966]

17. Zhuang Q et al., Graft-infiltrating host dendritic cells play a key role in organ transplant rejection. Nature Communications 7, Article number: 12623(2016).

18. Braza MS et al., Inhibiting inflammation with myeloid cell-specific nanobiologics promotes organ transplant acceptance. Immunity 49, 819-828 (2018). [PubMed: 30413362]

19. Batal I et al., Dendritic cells in kidney transplant biopsy samples are associated with T cell infiltration and poor allograft survival. J Am Soc Nephrol 26, 3102-3113 (2015). [PubMed: 25855773]

20. Takenaka K et al., Polymorphism in Sirpa modulates engraftment of human hematopoietic stem cells. Nat Immunol 8, 1313-1323 (2007). [PubMed: 17982459]

21. Pelletier S, Gingras S, Green DR, Mouse genome engineering via CRISPR-Cas 9 for study of immune function. Immunity 42, 18-27 (2015). [PubMed: 25607456]

22. Tun T et al., Genomic structure of mouse PIR-A6, an activating member of the paired immunoglobulin-like receptor gene family. Tissue antigens 61, 220-230 (2003). [PubMed: 12694571] 
23. Camirand $\mathrm{G}$ et al., Multiphoton intravital microscopy of the transplanted mouse kidney. Am J Transplant 11, 2067-2074 (2011). [PubMed: 21834913]

24. Corry RJ, Winn HJ, Russel PS, Primarily vascularized allografts of hearts in mice: The role of H-2D, H-2K, and non H-2 antigens. Transplantation 16, 343-350 (1973). [PubMed: 4583148]

25. Zheng GX et al., Massively parallel digital transcriptional profiling of single cells. Nat Commun 8 , 14049(2017). [PubMed: 28091601]

26. Qiu X et al., Single-cell mRNA quantification and differential analysis with Census. Nat Methods 14, 309-315 (2017). [PubMed: 28114287]

27. Trapnell $\mathrm{C}$ et al., The dynamics and regulators of cell fate decisions are revealed by pseudotemporal ordering of single cells. Nat Biotechnol 32, 381-386 (2014). [PubMed: 24658644]

28. Kanehisa M, Goto S, KEGG: kyoto encyclopedia of genes and genomes. Nucleic Acids Res 28, 27-30 (2000). [PubMed: 10592173] 


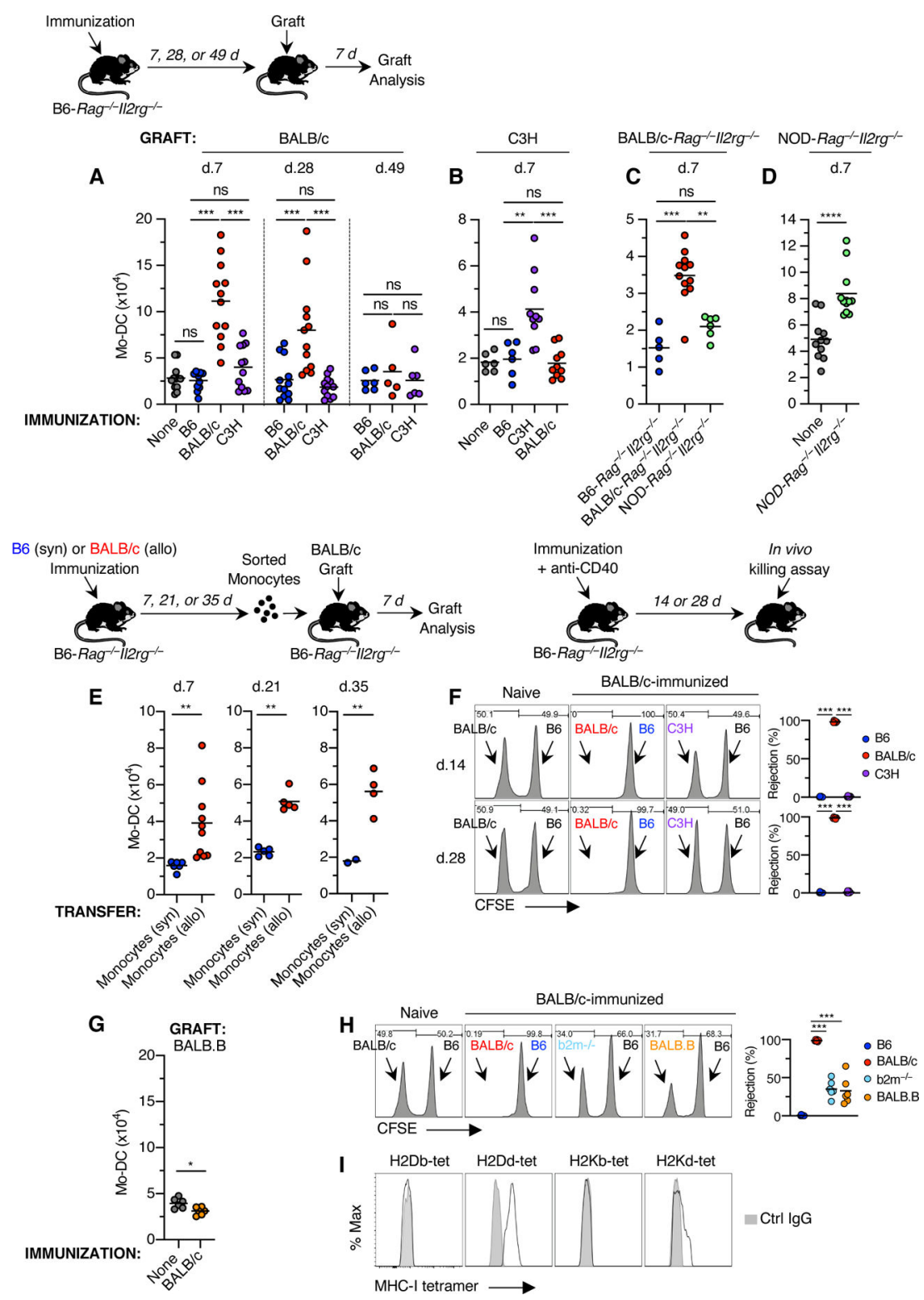

Fig. 1. Monocyte and macrophage memory specific to allogeneic MHC molecules.

A-D, Monocyte responses in immunized mice measured as monocyte-derived dendritic cells (Mo-DCs) in the graft ( $n=5$ to $12 / N=1$ to 2 ). $\mathbf{E}$, Monocytes from immunized mice transfer memory to naive recipients ( $n=2$ to $4 / N=1$ to 3 ). F, Allospecific killing of CFSE-labeled cells in immunized mice $(n=6 / N=2)$. $\mathbf{G}-\mathbf{H}$, Nonself MHC recognition is necessary for eliciting monocyte $(n=6 / N=1)(\mathrm{G})$ and macrophage $(n=6 / N=2)(\mathrm{H})$ memory. Killing of target cells lacking nonself MHC (BALB.B) or MHC-I (B2m $\left.{ }^{-/-}\right)$is significantly diminished (H). Assays were performed 7 days after immunizing B6- $\mathrm{Rag}^{-1-} I 12 \mathrm{rg}^{-1-}$ mice. I, Allogeneic MHC-I tetramer binding to macrophages from BALB/c-immunized B6- $\mathrm{Rag}^{-/} I I 2 \mathrm{rg}^{-/-}$hosts (representative of 3 experiments). Mean and individual biological replicates $(\mathrm{A}-\mathrm{H})$; one-way ANOVA (A-C, F, H) or two-tailed Student's $t$-test (D, E, G). 


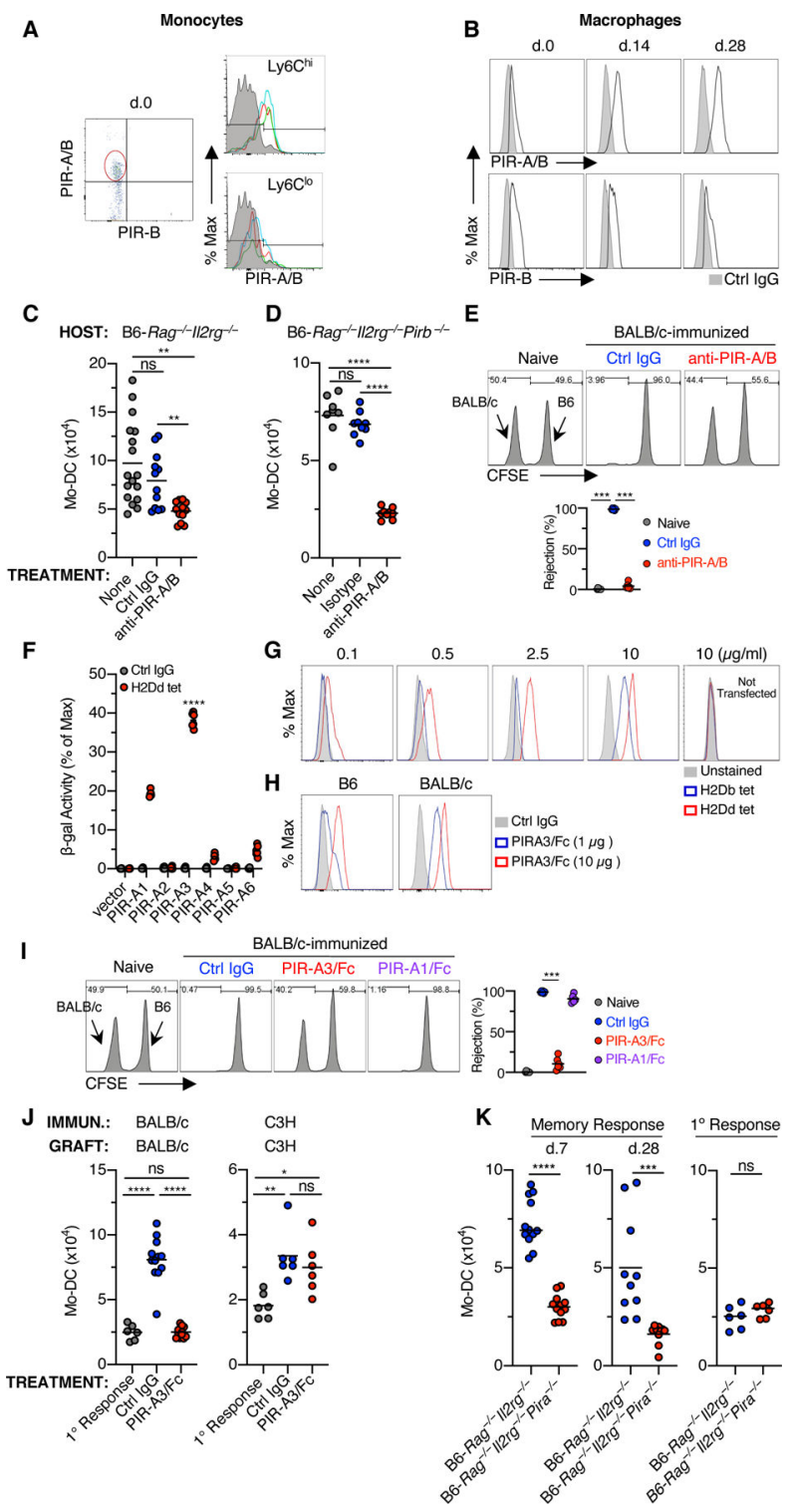

Fig. 2. Memory to allogeneic MHC-I is mediated by PIR-A molecules on monocytes and macrophages.

A-B, PIR-A and -B expression on Ly6C ${ }^{\text {hi }}$ monocytes (A) and macrophages (B) before (d.0) and after alloimmunization (d.14 \& d.28). Colored histograms $=$ biological replicates $(n=$

3). C-E, Blocking PIR-A/B inhibits monocyte $(n=12 / N=2)(\mathrm{C}-\mathrm{D})$ and macrophage $(n=6 /$ $N=2$ ) (E) memory. B6- $\mathrm{Rag}^{-/-} I 12 \mathrm{rg}^{-/-}$hosts were immunized with BALB/c splenocytes and rechallenged with BALB/c allografts 7 days later. F-H, H-2Dd MHC-I tetramers bind preferentially to PIR-A3-transfected BWZ.36 $(n=5 / N=1)(\mathrm{F})$ and 3 T3 cells (G). PIR$\mathrm{A} 3 / \mathrm{Fc}$ binds preferentially to $\mathrm{H}-2 \mathrm{Dd}^{+}(\mathrm{BALB} / \mathrm{c})$ cells $(\mathrm{H})$. I-J, Specific inhibition of macrophage $(n=5-6 / N=2)(\mathrm{I})$ and monocyte $(n=6 / N=1$ to 2$)(\mathrm{J})$ memory to BALB/c allografts by PIR-A3/Fc. K, Absent memory 7 and 28 days after immunization, but normal primary monocyte alloresponse in Pira $^{-/}$hosts $(n=6 / N=1$ to 2$)$. Representative of three experiments $(\mathrm{B}, \mathrm{G}-\mathrm{H})$; mean and individual biological replicates $(\mathrm{C}-\mathrm{D}, \mathrm{F}, \mathrm{I}-\mathrm{K})$; one-way ANOVA (C-E, I-J) or two-tailed Student's $t$-test (K). 
A
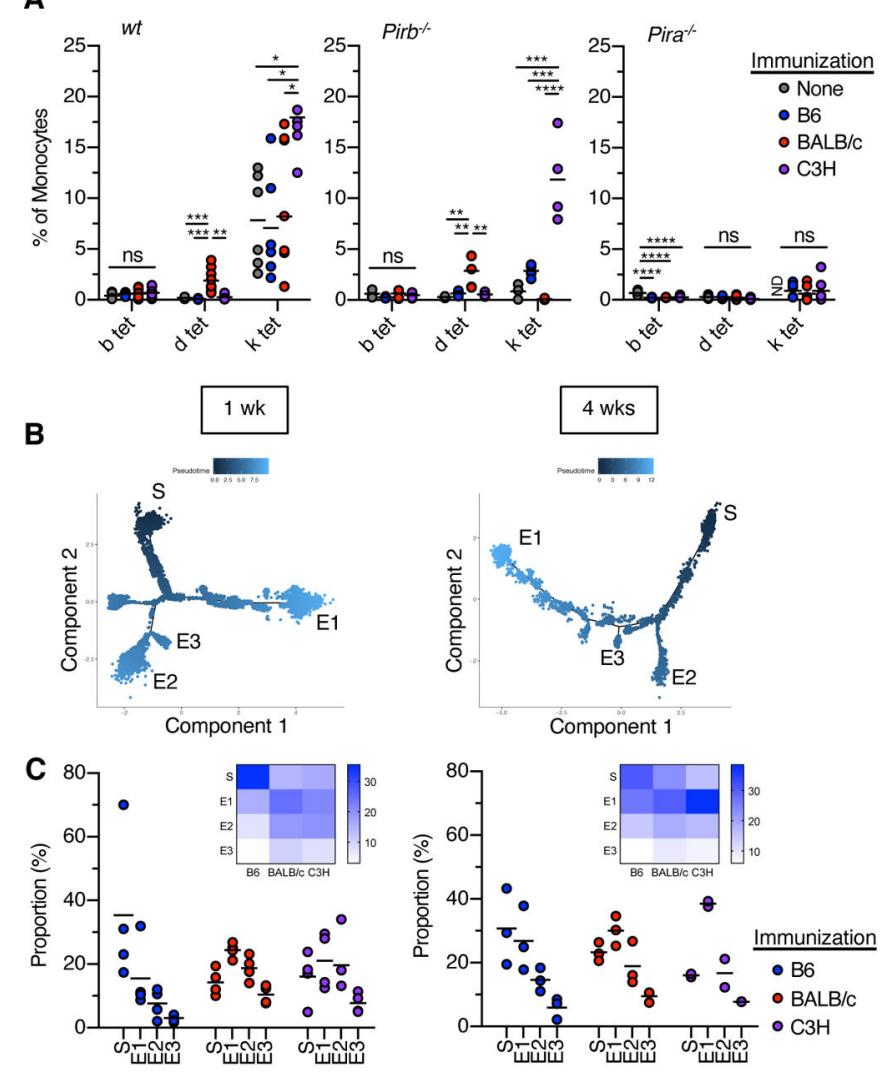

D
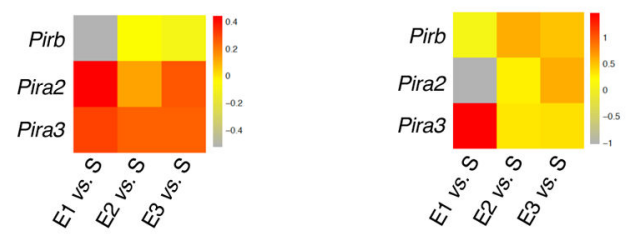

E
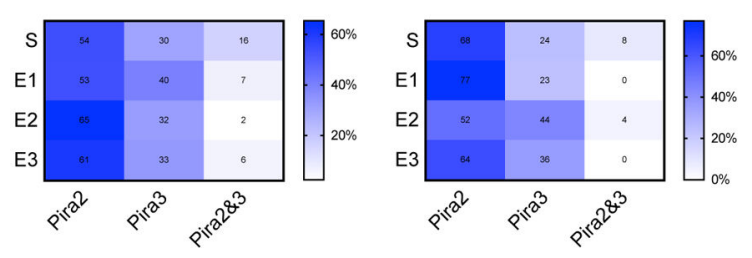

Fig. 3. Mechanisms of monocyte memory.

A, Tetramer-positive splenic monocytes 7 days after immunizing B6- $\mathrm{Rag}^{-1-} \mathrm{II2}^{-\mathrm{rg}^{-1}}$ mice (mean and individual biological replicates; $n=3 / N=1$ to 3 ). B-E, Splenic monocyte scRNA-seq analysis 1 and 4 weeks post-immunization $(n=3 / N=1)$ : Pseudotime plots, $\mathrm{S}=$ Starting state, E1-3 = Expanding states (B); heatmaps and graphs depicting proportion of $S$ and E states in each immunization group (C); differential expression of Pir genes in E states vs. S state (D); and proportion of monocytes expressing Pira2, Pira3, or both in each state (E). one-way ANOVA (A). 
A

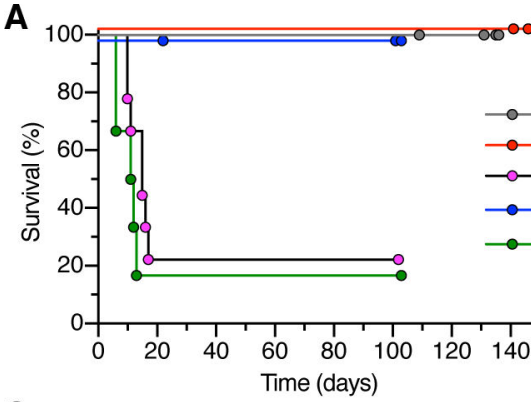

C

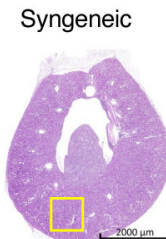

岸

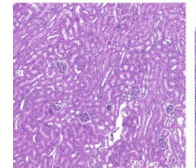

wt

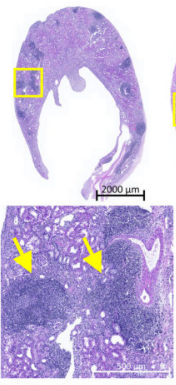

Pira-

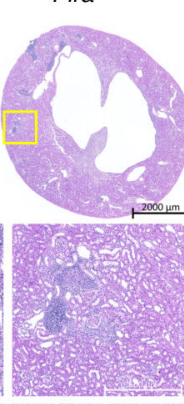

Pirb--

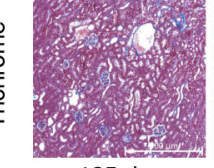

$>125 d$

E

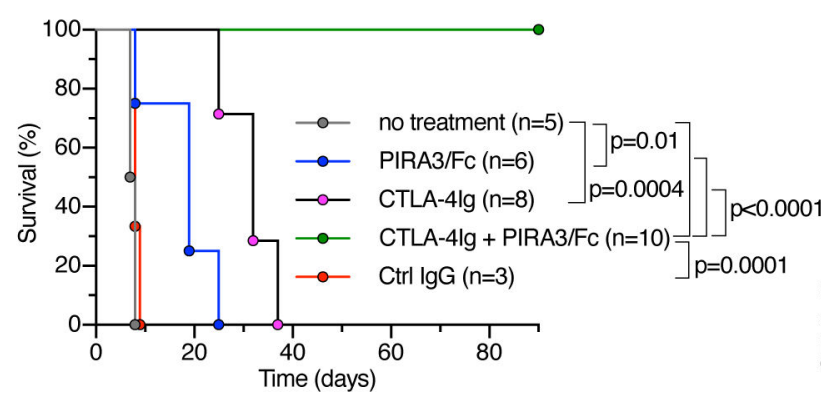

B
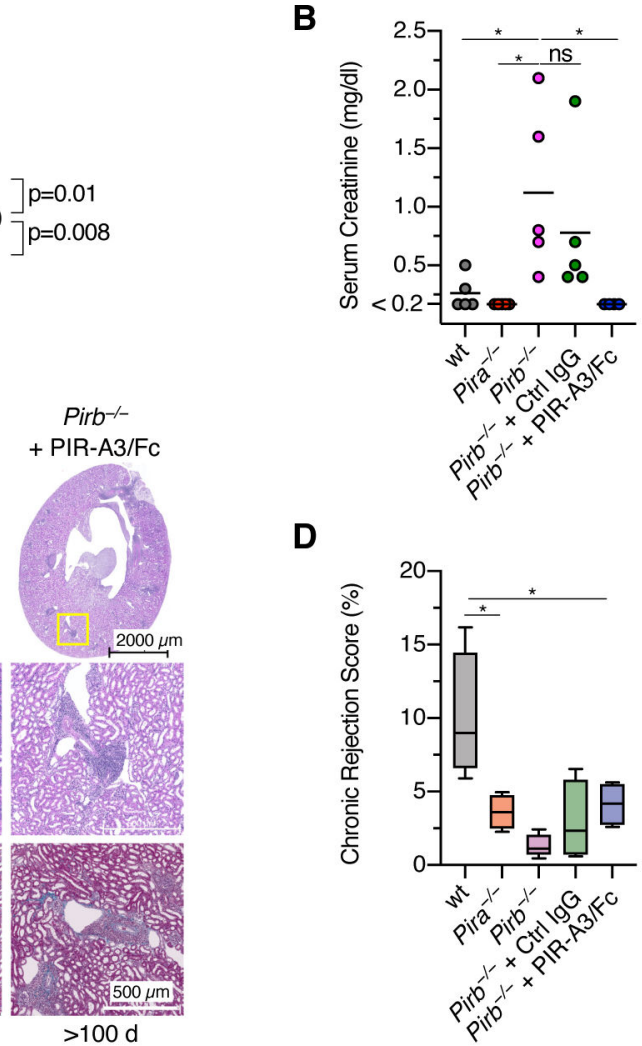

F
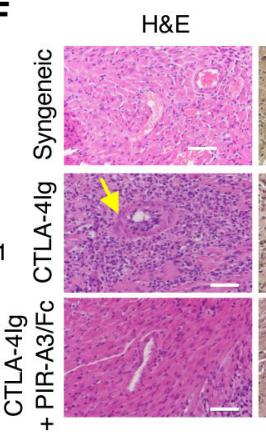

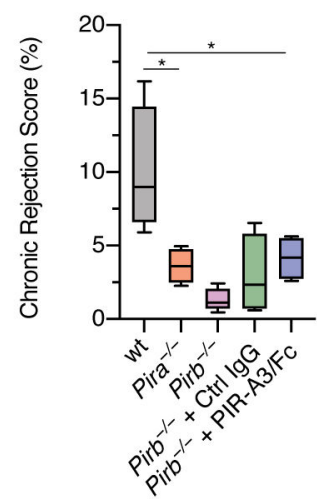

VVG

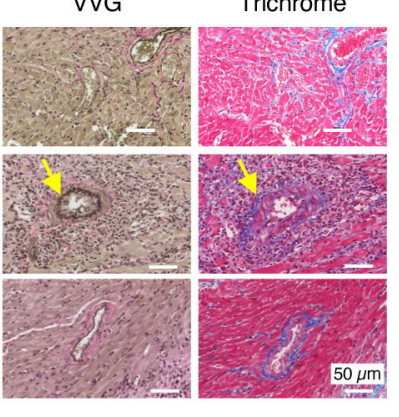

Fig. 4. Genetic deletion or blocking PIR-A attenuates kidney and heart allograft rejection.

A-D, Survival (A), serum creatinine (B), histology (C), and chronic rejection scores (D) of BALB/c kidneys transplanted to wildtype (wt), Pira $^{-1-}$ or Pirb $^{-1-}$ B6 recipients. Arrows point to infiltrates and fibrosis. E-F, Survival (E) and histology (F) of BALB/c hearts transplanted to B6 mice treated with PIR-A3/Fc ( \pm CTLA4Ig) or control mouse IgG1 (Ctrl IgG). Arrows point to chronic rejection vasculopathy. Mean and individual biological replicates (B, D); $\log$-rank (A, E) and one-way ANOVA (B, D). 